\title{
Correlation of Radiologic and Pathologic Response in Patients Receiving Neoadjuvant Radiotherapy for Soft Tissue Sarcoma
}

\author{
Ramiz Abu Hijlih*1, Sara Mheid' ${ }^{1}$, Fawzi Abuhijla1, Wafa Asha1, Issa Mohamad', Sameer Yaser², Ahmed Shehadeh ${ }^{3}$, \\ Samer Abdal $^{3}$, Abdelatif Almousa ${ }^{1}$ and Samer Salah MD $^{2}$ \\ ${ }^{1}$ Radiation Oncology department, King Hussein Cancer Center, Jordan \\ ${ }^{2}$ Medical Oncology department, King Hussein Cancer Center, Jordan \\ ${ }^{3}$ Department of surgery, King Hussein Cancer Center, Jordan
}

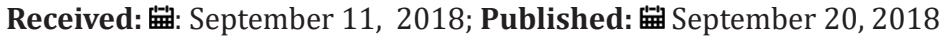

*Corresponding author: Ramiz Abu-Hijlih MD, Radiation Oncology department, King Hussein Cancer Center, Amman-Jordan

\begin{abstract}
Background: It is unknown if the radiologic response after neoadjuvant radiation (RT) in patients with soft tissue sarcoma correlates with the histologic response or disease outcomes. The purpose of this study is to evaluate radiographic and histologic responses in patients who received neoadjuvant RT, and to evaluate the relation between these short-term surrogates.

Patients and Methods: We sought to review consecutive patients with primary localized STS, who were treated with preoperative RT, between 2016 and 2018 at a single institution. All patients were required to have initiated RT with the intention to be followed by curative surgical resection. Data on demographics and disease characteristics were retrospectively collected, the tumor volume (cc) was calculated before and after RT, pathology specimens were reviewed for percent of necrosis and status of surgical margins.
\end{abstract}

Results: Twenty-three patients with primary localized STS were treated with preoperative radiotherapy followed by surgery. There were 11 extremity (48\%), 7 retroperitoneal (30\%) and 5 thoracic (22\%) tumors. The tumor volume decreased after neoadjuvant RT in 10 cases $(43 \%)$ to a maximum of $50 \%$ (range, -5 to $-50 \%$ ), while in eight patients (35\%) the tumor grew in size to a maximum of $40 \%$ (range, +10 to $+40 \%$ ). The tumor volume was stable in five patients (22\%). Complete resection (R0) was achieved in 18 cases (79\%), microscopically involved margins (R1) were observed in $4(17 \%)$, and gross residual (R2) in one patient (4\%). The median tumor necrosis was 40\%. Five patients (22\%) demonstrated complete or near-complete pathologic response ( $\geq 95 \%$ necrosis). Pearson correlation coefficient test revealed no correlation between radiologic and histologic responses (- 0.07). Major wound complication after surgery was observed in four patients (17\%).

Conclusion: Radiologic response after neo-adjuvant RT was a poor predictor of pathologic response. Complete and near complete histologic responses seem to be associated with favorable clinical outcomes. Larger studies are needed to test these surrogates in a prospective fashion.

Abbreviations: CT: Computed Tomography; MLS: Myxoid Liposarcoma; MRI: Magnetic Resonance Imaging; IRB: Institutional Review Board; CTV: Clinical Target Volume; PTV: Planning Target Volume; IMRT: Intensity Modulated Radiotherapy; VMAT: Volumetric Modulated Arc Therapy; DFS: Disease Free Survival; OS: Overall Survival

\section{Introduction}

Soft tissue sarcomas are exceedingly rare tumors that account for $1 \%$ of all human malignancies. More than 50 histologic subtypes of STS are recognized. Limb salvage surgery and radiation (RT) are the standard of care in management of extremity sarcomas [1]. The timing of RT; whether preoperative or postoperative, has been tested in a prospective trial [2], both approaches have comparable oncologic outcomes in terms of local control and survival. However, preoperative RT was found to have a higher risk of major wound complications. On the contrary, late side effects such as fibrosis, joint stiffness and edema were more prevalent in patients receiving postoperative RT [3]. Soft tissue sarcoma volume usually changes after neoadjuvant RT. Many physicians perceive this response as a sign for disease response and treatment outcome. However, this suggestion was not studied nor proven. Another surrogate is post-treatment tumor necrosis; the effect of histologic response on disease outcome is not well defined and data are conflicting.

Nevertheless, some studies have reported better survival with near complete necrosis [4]. Furthermore, it is unknown if radiologic response does correlate with pathologic response in the surgical specimen following neoadjuvant RT. The aim of this study is to 
evaluate tumor volume changes after preoperative RT and to assess tumor necrosis on histologic examination. Furthermore, we aim to test the correlation between radiologic and histologic response.

\section{Materials and Methods}

\section{Patient population}

Between January 2016 and July 2018, twenty-three patients aged $\geq 18$ years were diagnosed with soft tissue sarcoma at KHCC. An experienced musculoskeletal pathologist reviewed the pathology specimens. Staging work-up included bone scan and chest Computed Tomography (CT) scan. Abdominal CT scans were added for patients with myxoid liposarcoma (MLS) or whenever indicated by patients symptoms. Magnetic Resonance Imaging (MRI) was performed to assess the tumor before and after RT. Institutional Review Board (IRB) at KHCC approved this study and the data was retrospectively collected. We analyzed the RT plans, imaging and pathology reports. Data was collected on patients' age, sex, tumor location and volume, in addition to information on local recurrence and survival. Treatment side effects were also quantified, in particular, major wound complications, which was defined according to NCIC-SR2 trial; secondary operation under general or regional anesthesia for wound repair or management [2].

\section{Treatment procedure}
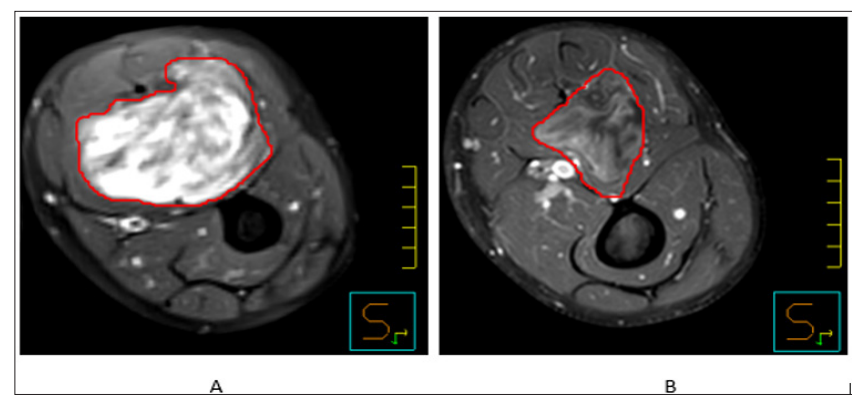

Figure 1: depicts MRI images for thigh soft tissue sarcoma, uploaded to Pinnacle; a. GTV delinated before RT. B. GTV contour after RT, with good partial response.

Before starting treatment, all cases were discussed at the multidisciplinary tumor boards. We only included patients who were managed by preoperative RT as a monotherapy. CT-simulation was performed for all the patient cohort. MRI images (T1 weighted, post gadolinium and T2- weighted) were uploaded to Pinnacle system Version 9.6 (Koninklijke Philips N.V., Amsterdam, Netherlands), then fused with the simulation scan, and registered to match the bony and soft tissue anatomy. Then Gross Target Volume was delineated using both images, the Clinical Target Volume (CTV) and Planning Target Volume (PTV) were created following RTOG 0630 protocol [5]. Intensity Modulated Radiotherapy (IMRT) and Volumetric Modulated Arc Therapy (VMAT) techniques were used for planning. The total RT dose was 50Gy, administered in 2-Gy fractions over 25 sessions. New MRI was obtained one month after completion of RT. Post-operative MRI was also uploaded to Pinnacle, where the tumor was delineated and the volume was calculated again (Figure 1). In this cohort we did not rely on Response Evaluation Criteria in Solid
Tumors RECIST response criteria since it takes into account only one dimension. Furthermore, based on previous reports, RECIST response was found to be a poor prognostic marker of disease outcomes [6-8]. For these reasons, we did a pathologic correlation with the exact tumor volume rather than use the RECIST response criteria.

Surgical resection was typically performed 4-8 weeks after completion of RT. Surgeries were performed on the intention to achieve complete (R0 resection) for all patients. All operative pathologic specimens were assessed for completeness of resection (margin status) and the percent tumor necrosis. The degree of tumor resection was graded by operative reports and post-operative contrast enhanced MRI; complete resection (R0), microscopic residual (R1) and gross residual (R2).

\section{Statistical Analysis}

The overall survival (OS) was defined as time from primary diagnosis to time of death from any cause. The disease-free survival (DFS) was calculated from time of diagnosis to time of first evidence of local recurrence or distant metastasis, whichever comes first. The Pearson correlation coefficient was used to test the correlation between radiologic response and percent of necrosis. Survival was estimated by the Kaplan-Meier and compared between groups by the log-rank test [9]. All p values $\leq 0.05$ were considered statistically significant. All analyses were performed using SAS version 9.4 (SAS Institute Inc, Cary, NC).

\section{Results}

\section{Patient and Tumor Characteristics}

Twenty-three adult patients received neoadjuvant RT for soft tissue sarcoma with radical intent. Most of them were men 15 (66\%). The median age was 43 years (range, 19-78). Major histologic subtypes were MLS and dedifferentiated Liposarcoma, 6 cases each (26\%). The site of primary tumor was most commonly the lower extremity (11 cases; 48\%); followed by the retroperitoneum (7 cases; $30 \%$ ). All tumors, except one, were deep to the fascia. The mean tumor volume was $810 \mathrm{cc}$ (range, 55-4000). There was one patient with thigh MLS and a solitary abdominal metastatic lesion, and the metastatic mass was resected with curative intent before starting radiation to the thigh. All other patients had non-metastatic at time of diagnosis. Table 1 illustrates patients and disease characteristics. Seven patients $(30 \%)$ with retroperitoneal tumors underwent laparotomy and resection of the tumor. Interestingly, $\mathrm{R} 1$ resections were more prevalent in this group and three cases had a positive resection margin. On analysis, these tumors were larger than other sites, with a median volume of $1450 \mathrm{cc}$ (range, 90-2850). During surgery, these lesions were frequently infiltrative, and entailed removal of the ipsilateral kidney and colon. In patients with extremity primary tumor, all resections were complete, and only three had close surgical margin (less than $10 \mathrm{~mm}$ ). Amputation was inevitable in one patient with a huge tumor encasing the main vascular bundle. The main treatment adverse effects were; wound infection, drop foot and pneumothorax. Major wound complications (as defined per NCIC-SR2 trial) were observed in four cases (18\%), all of them were lower extremity location. 
Table 1: Patient and disease characteristics.

\begin{tabular}{|c|c|}
\hline Age at diagnosis (yr) & Median 43 (19-78) \\
\hline Gender, n (\%) & Male-15 (66) \\
Tumor Location, n (\%) & Lower extremity- 11 (48) \\
Retroperitoneal- 7 (30) \\
Chest wall- 5 (22)
\end{tabular}

\section{Treatment Outcome}

After a median follow-up of 12 months (range, 4-31), twenty one patients are still alive (91\%). Local recurrence was documented in four patients, three of them had positive surgical margins and three were retroperitoneal tumors. Intriguingly, three were associated with distant metastasis. At time of last analysis two were dead. In total, distant metastases were depicted in nine patients (eight in the lung and one in the abdomen). Chemotherapy was prescribed for all patients in relapse (mainly Adriamycin and Ifosfamide-IA). Furthermore, metastatectomy was performed for five patients with resectable lung metastasis. At time of last follow-up, 18 patients (78\%) were alive without evidence of disease and three were alive with disease. Treatment characteristics and disease outcome are shown in Table 2.

Table 2: Treatment characteristics and disease outcomes.

\begin{tabular}{|c|c|}
\hline \multirow{2}{*}{ Volume change after RT, $\mathrm{n}(\%)$} & $\begin{array}{c}\text { Decrease- } 10(45) \\
\text { The same- } 5(21) \\
\text { Increase- 8 }(34\end{array}$ \\
\hline \multirow{2}{*}{ Type of surgery, n (\%) } & Wide local excision- 15 (66) \\
& Amputation-1 (4) \\
Laparotomy-7 (30)
\end{tabular}

\begin{tabular}{|c|c|}
\hline \multirow{3}{*}{ Status at last follow-up, n (\%) } & No evidence of disease-18 (78) \\
& Alive with disease- 3 (13) \\
Dead of other causes- 0 \\
Dead of disease- 2 (9)
\end{tabular}

\section{Correlation of Radiologic and Pathologic Response}

Post-RT imaging revealed that tumor volume had changed in 18 cases $(78 \%)$, and it was stable only in five patients. Tumor growth was observed in eight patients (34\%), the median change was $21 \%$ (range, +10 to $+40 \%$ ). While disease regression was documented in 10 cases (45\%), median shrinkage was $28 \%$ (range, -10 to $-50 \%$ ). (Figure 2) illustrates the distribution of these responses. Tumor necrosis was a common finding on specimen examination; the median pathologic necrosis was 40\% (range, 10-100\%). Complete and near-complete responses $(\geq 95 \%)$ were observed in five cases $(22 \%)$. Presence of necrosis $>95 \%$ was associated with favorable disease outcomes, local control 100\%, and survival 100\%. However, when patients with necrosis ( $>95 \%)$ were compared with those with $(<95 \%)$ necrosis, there was no statistical significant difference in any of the oncologic endpoints (Figure 3). Both tumor radiologic progression and disease regression did not correlate with the percent of necrosis $(\mathrm{p}=0.517)$. Pearson correlation coefficients showed no correlation between the two parameters (- 0.07). Pathologic complete response was more frequent in MLS subtype, three patients showed complete or near complete response, and they are alive without evidence of disease.

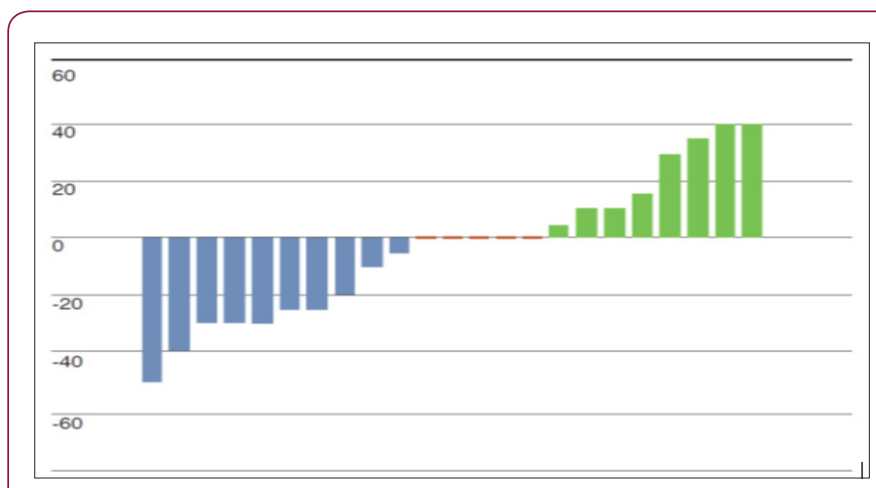

Figure 2: Waterfall plot demonstrates radiographic response percentages after neoadjuvant RT.

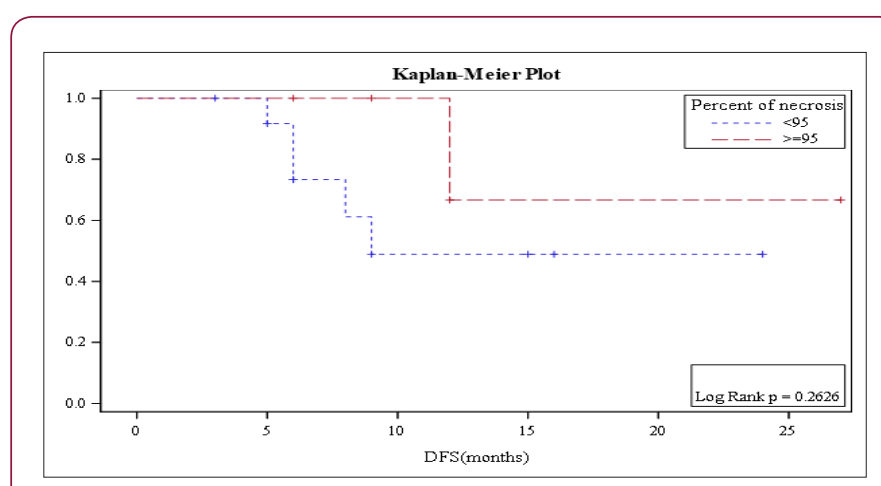

Figure 3: Kaplan-Meier disease free survival estimation by extent of pathologic necrosis. 


\section{Discussion}

The primary therapy for soft tissue sarcomas is surgical resection. RT is an important component of the multimodality treatment, contributing substantially to both local control and limb-salvage surgery results. Neoadjuvant RT gives the opportunity to assess the response to therapy using radiological, pathological and clinical parameters [10-12]. O'Sullivan et al evaluated the use of image guided IMRT in a prospective phase II trial, and they found the incidence of major wound complications lower when this technique was used [13]. Moreover, RTOG 0630 utilized imageguided IMRT with smaller margins around the tumor, resulting in significant reduction in late toxicity without marginal recurrences [5]. Clinical use of new radiation techniques, such as IMRT, is increasing worldwide [14], along with the utilization of image guidance. These advancements necessitate understanding of the significance of tumor volume changes during and after RT, and how such changes correlate with the percent of necrosis and oncologic outcomes. Only a few reports in the literature tested the correlation between these short-term surrogates. Canter et al evaluated radiologic and histologic correlation in 25 patients.

Interestingly none of their patient demonstrated radiologic response, although nine patients (36\%) showed tumor necrosis $(\geq 50 \%)$ and two with near-complete response. They concluded that near complete responses are rare events that may indicate better outcomes, and that there was no correlation between histologic response and RECIST response [15]. Our results came in concordance with this conclusion; radiologic response is not a reliable marker for histologic response or disease outcome. Radiologic pseudo-progression seems to be a common finding after neoadjuvant RT, and it may result from reactive edema or tumor liquefaction inside the capsule. Novel and functional imaging may help to detect true progression [16]. Furthermore, tumor progression does not appear to affect resectability or surgical outcome. A review on 68 patients with borderline resectable extremity and trunk sarcoma showed that tumor progression had no effect on surgical outcomes or local control rates [17].

Percent of necrosis has been suggested as a short-term surrogate. However, results from previous studies were contradicting; Willett et al and Hew et al did not demonstrate a correlation between necrosis greater than $80 \%$ and distant metastasis free survival $[18,19]$. Nevertheless, other reports showed improvement of survival in patients with near complete necrosis when chemotherapy was administered with neoadjuvant RT $[20,21]$. In our series, we observed a median necrosis of $40 \%$ and near-complete response in $22 \%$ of patients, which is lower than previous reports (29$58 \%$ ), and this fact may be related to the addition chemotherapy. Recently, Salah et al performed a meta-analysis on 1663 patients, and demonstrated a statistical significant improvement in disease free survival and overall survival with attainment of $\geq 90 \%$ necrosis following various neoadjuvant modalities. Noteworthy the benefit was not observed in patients who received RT as a monotherapy [4]. MLS is a highly radiosensitive tumor, and many reports suggest favorable local control and survival in this subset of patients. In addition, the percent of necrosis is found to be higher. Our results support these findings; nevertheless, we did not find a statistically significant difference between MLS and the other histologic subtypes, most likely owing to the small sample size.

Only a few studies in the literature tested the correlation between the radiologic and histologic outcomes, and this work will be added to the current body of evidence. The limitations of this study are the retrospective nature and associated selection bias. In addition, the study was not powered to detect a statistical significant difference; which raises questions on the external validity of the results. When we evaluated the major wound infection in lower extremity location, the incidence rate was $36 \%$, which is close to the previous trials which used IMRT techniques $[5,13]$.

\section{Conclusion}

Soft tissue sarcoma volume usually change after neoadjuvant RT. Tumor progression is not uncommon, and it may represent reactive edema and tumor liquefaction or it may denote a true tumor progression. Tumor necrosis after neoadjuvant RT has been suggested as a surrogate for disease outcome. The primary aim of this study is to assess correlation between radiologic and histologic response; and we did not find any relation between both responses.

\section{References}

1. Rosenberg SA, Tepper JE, Glatstein EJ, J Costa, A Baker, et al. (1982) The treatment of soft tissues arcomas of the extremities: prospective randomized evaluations of (1) limb-sparing surgery plus radiation therapy compared with amputation and (2) the role of adjuvant chemotherapy. Ann Surg 196(3): 305-315.

2. Sullivan B, Davis AM, Turcotte R, Bell R, Catton C, et al. (2002) Preoperative versus postoperative radiotherapy in soft-tissue sarcoma of the limbs: a randomised trial. Lancet 359(9325): 2235-2241.

3. Davis AM, O Sullivan B, Turcotte R, Bell R, Catton C, et al. (2005) Late radiation morbidity following randomization to preoperative versus postoperative radiotherapy in extremity soft tissue sarcoma. Radiother Oncol 75(1): 48-53.

4. Salah S, Lewin J, Amir E, Abdul Razak A (2018) Tumor necrosis and clinical outcomes following neoadjuvant therapy in soft tissue sarcoma: A systematic review and meta-analysis. Cancer Treat Rev 69: 1-10.

5. Wang D, Zhang Q, Eisenberg BL, Kane JM, Li XA, et al. (2015) Significant Reduction of Late Toxicities in Patients with Extremity Sarcoma Treated With Image-Guided Radiation Therapy to a Reduced Target Volume: Results of Radiation Therapy Oncology Group RTOG-0630 Trial. J Clin Oncol 33(20): 2231-2238.

6. Stahl R, Wang T, Lindner LH, Abdel Rahman S, Santl M, et al. (2009) Comparison of radiological and pathohistological response to neoadjuvant chemotherapy combined with regional hyperthermia (RHT) and study of response dependence on the applied thermal parameters in patients with soft tissue sarcomas (STS). Int J Hyperthermia 25(4): 289-298.

7. Benjamin RS (2008) SARC-CTOS imaging symposium: introduction to the problem from a clinical perspective. Oncologist 13(Suppl 2): 1-3.

8. Ueda $\mathrm{T}$, Naka N, Araki N, et al. (2008) Validation of radiographic response evaluation criteria of preoperative chemotherapy for bone and soft tissue sarcomas: Japanese Orthopaedic Association Committee on Musculoskeletal Tumors Cooperative Study. J Orthop Sci 13(4): 304-312.

9. Kaplan EL, Meier P (1958) Nonparametric estimation from incomplete observations. J Am Stat Assoc 53(282): 457-481. 
10. Lewis JJ, Brennan MF (1996) Soft tissue sarcomas. Curr Probl Surg 33(10): 817-872.

11. Wunder JS, Nielsen TO, Maki RG, O'Sullivan B, Alman BA (2007) Opportunities for improving the therapeutic ratio for patients with sarcoma. Lancet Oncol 8(6): 513-524.

12. Pisters PW, O Sullivan B, Maki RG (2007) Evidence-based recommendations for local therapy for soft tissue sarcomas. J Clin Oncol 25(8): 1003-1008.

13. Sullivan B, Griffin AM, Dickie CI, Sharpe MB, Chung PW, et al. (2013) Phase 2 Study of Preoperative Image-Guided Intensity-Modulated Radiation Therapy to Reduce Wound and Combined Modality Morbidities in Lower Extremity Soft Tissue Sarcoma. Cancer 119(10): 1878-1884.

14. Mell LK, Mehrotra AK, Mundt AJ (2005) Intensity modulated radiation therapy use in the US, 2004. Cancer 104(6): 1296-1303.

15. Canter R, Martinez S, Tamurian R, Wilton M, Li CS, et al. (2010) Radiographic and Histologic Response to Neoadjuvant Radiotherapy in Patients With Soft Tissue Sarcoma. Ann Surg Oncol 17(10): 2578-2584.

16. Benz MR, Czernin J, Allen Auerbach MS, Tap WD, Dry SM, et al. (2009) FDG-PET/CT imaging predicts histopathologic treatment responses after the initial cycle of neoadjuvant chemotherapy in high-grade softtissue sarcomas. Clin Cancer Res 15(8): 2856-2863.

17. le Grange F, Cassoni AM, Seddon BM (2014) Tumour volume changes following pre-operative radiotherapy in borderline resectable limb and trunk soft tissue sarcoma. Eur J Surg Oncol 40(4): 394-401.

18. Willett CG, Schiller AL, Suit HD, Mankin HJ, Rosenberg A, et al. (1987) The histologic response of soft tissue sarcoma to radiation therapy. Cancer 60(7): 1500-1504.

19. Hew L, Kandel R, Davis A, O Sullivan B, Catton C, et al. (1994) Histological necrosis in soft tissue sarcoma following preoperative irradiation. J Surg Oncol 57(2): 111-114.

20. Macdermed DM, Miller LL, Peabody TD, et al. (2010) Primary Tumor Necrosis Predicts Distant Control in Locally Advanced Soft-Tissue Sarcomas After Preoperative Concurrent Chemoradiotherapy. Int J Radiat Oncol Biol Phys 76(4): 1147-1153.

21. Eilber FC, Rosen G, Eckardt J, et al. (2001) Treatment-induced pathologic necrosis: a predictor of local recurrence and survival in patients receiving neoadjuvant therapy for high-grade extremity soft tissue sarcomas. J Clin Oncol 19(13):3203-3209.
ISSN: 2574-1241

DOI: 10.26717/BJSTR.2018.09.001765

Ramiz Abu-Hijlih MD. Biomed J Sci \& Tech Res

This work is licensed under Creative Commons Attribution 4.0 License

Submission Link: https://biomedres.us/submit-manuscript.php

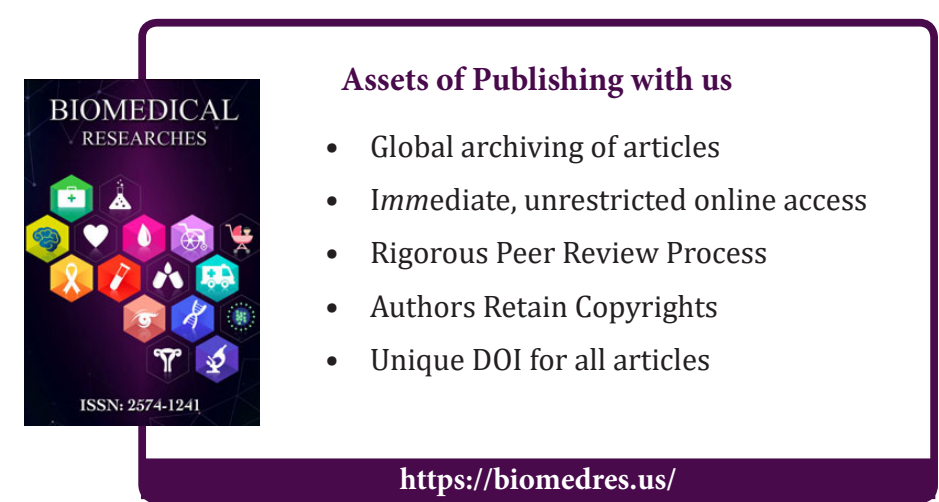

